\title{
CircHIPK2 Contributes to DDP Resistance and Malignant Behaviors of DDP-Resistant Ovarian Cancer Cells Both in vitro and in vivo Through circHIPK2/miR-338-3p/CHTOP ceRNA Pathway
}

\author{
Yang Cao' \\ $\mathrm{Xin} \mathrm{Xie}^{2}$ \\ Mingzhu $\mathrm{Li}^{3}$ \\ Yuhua Gao' \\ 'Department of Gynaecology, Cancer \\ Hospital of China Medical University, \\ Liaoning Cancer Hospital \& Institute, \\ Shenyang City, Liaoning Province, \\ People's Republic of China; ${ }^{2}$ Department \\ of Teaching and Research Center, \\ Liaoning University of Traditional Chinese \\ Medicine, Shenyang City, Liaoning \\ Province, People's Republic of China; \\ ${ }^{3}$ Department of Integrated Traditional \\ Chinese and Western Medicine Medical \\ Oncology, Cancer Hospital of China \\ Medical University, Liaoning Cancer \\ Hospital \& Institute, Shenyang City, \\ Liaoning Province, People's Republic of \\ China
}

Background: Cisplatin (DDP) is standard-of-care and first-line management for ovarian cancer (OvCa). Circular RNA HIPK2 (circHIPK2) is abnormally upregulated in serum of OvCa patients. However, its role in DDP resistance remains unclear.

Methods: Expression of cirHIPK2, microRNA (miR)-338-3p and chromatin target of protein arginine methyltransferase (CHTOP) was detected by quantitative reverse transcription PCR and Western blotting. Functional experiments were performed using cell counting kit- 8 assay, flow cytometry, transwell assays, Western blotting, and xenograft experiment. The interaction among cirHIPK2, miR-338-3p and CHTOP was confirmed by dual-luciferase reporter assay and RNA pull-down assay.

Results: Expression of circHIPK2 and CHTOP was upregulated, and miR-338-3p was downregulated in human DDP-resistant OvCa tumors and cells. Blocking circHIPK2 could promote apoptosis and suppress the 50\% inhibitory concentration (IC50) of DDP, cell proliferation, cell cycle entrance, migration and invasion in SKOV3/DDP and A2780/DDP cells. Allied with that was decreased B cell lymphoma (Bcl)-2, matrix metalloproteinase 2 (MMP2) and MMP9 levels, and increased Bcl-2-associated X protein (Bax) level. Similarly, overexpression of miR-338-3p functioned suppressive role in SKOV3/DDP and A2780/DDP cells. MiR-338-3p was a target for circHIPK2, and CHTOP was targeted by miR-338-3p, whereas silencing miR-338-3p counteracted the role of circHIPK2 knockdown, and restoring CHTOP either cancelled miR-338-3p role. The growth of A2780/DDP cells in nude mice was restrained by silencing circHIPK2 under DDP treatment or not.

Conclusion: CircHIPK2 might be a tumor promoter in OvCa and was associated with DDP resistance. Silencing circHIPK2 might suppress DDP-resistant OvCa through regulating miR-338-3p/CHTOP axis.

Keywords: circHIPK2, miR-338-3p, ovarian cancer, DDP resistance, CHTOP

\section{Introduction}

Ovarian cancer $(\mathrm{OvCa})$ is one of the top gynecological malignancies, and the epithelial type accounts for $90 \%$ of primary $\mathrm{OvCa}^{1,2} \mathrm{OvCa}$ is extremely heterogeneous, ${ }^{3}$ and contains four subtypes, including serous, endometrioid, clear cell, and mucinous according to World Health Organization (WHO) classification system. Platinum-based adjuvant chemotherapy combined with taxane is the standard-of-care and first-line management for $\mathrm{OvCa}$ patients after cytoreductive
Department of Gynaecology, Cancer Hospital of China Medical University, Liaoning Cancer Hospital \& Institute, No. 44 Xiaoheyan Road, Shenyang, II 0042, Liaoning Province, People's Republic of China

$\mathrm{Tel} / \mathrm{Fax}+86024-31916913$

Email gaol594@I26.com 
surgery. ${ }^{4}$ Even though OvCa is cisplatin (DDP)-responsive tumor, ${ }^{5}$ acquired chemoresistance to platinum-containing drugs invariably happens in $\mathrm{OvCa}$ patients within 6 months of DDP treatment. ${ }^{6}$ Thereby, it is important to investigate the molecular mechanism and development of DDP resistance in OvCa.

Emerging roles of informative noncoding RNAs have been highlighted in regulating DDP resistance, such as long noncoding RNAs (lncRNAs), ${ }^{7}$ microRNAs (miRNAs), ${ }^{8}$ and circular RNAs (circRNAs). ${ }^{9}$ CircRNAs are covalently closed, endogenous RNAs that have crucial biological functions in human diseases with tissue-specific and cell-specific patterns. ${ }^{10}$ The expression, function and mechanism of circRNAs have been systematically overviewed in gynecological cancers including OvCa. ${ }^{11}$ Homeodomain-interacting protein kinase-2 (HIPK2), a key regulator of DNA damage response and epigenome, ${ }^{12}$ could splice a circular HIPK2 (circHIPK2; ID: hsa_circ_0001756) from the exon 2. Moreover, DDP is a DNA-damaging agent. ${ }^{5}$ Thus, we wondered whether circHIPK2 is essential in DDP resistance.

CircRNAs could sponge diverse miRNAs to mediate a pivotal competing endogenous RNA (ceRNA) regulatory network which plays an important role in tumorigenesis and DDP resistance. ${ }^{13,14}$ MiR-338-3p is a well-recognized participator and regulator in tumorigenesis and DDP resistance of $\mathrm{OvCa},{ }^{15,16}$ as well as chromatin target of protein arginine methyltransferase (CHTOP). ${ }^{17,18}$ In this study, we investigated the role of circHIPK2 in DDP resistance and malignant behaviors of OvCa cells acquired DDP resistance, as well as the potential ceRNA pathway among circHIPK2, miR-338-3p and CHTOP.

\section{Materials and Methods}

\section{Human Tissue Collection}

A sum of $46 \mathrm{OvCa}$ tissues and adjacent healthy tissues were acquired from Cancer Hospital of China Medical University, Liaoning Cancer Hospital \& Institute. All those patients received no chemotherapy or radiotherapy prior to this operation; 30 cases were diagnosed with primary epithelial $\mathrm{OC}$, and 16 cases were endometrioid OvCa; other clinicopathological characteristics of all subjects are shown in Table 1. After the last DDP-based treatment, these OvCa tumors were divided into DDPsensitive ones $(n=26)$ and DDP-resistant ones $(n=20)$. All patients and their families agreed to participate in the study and signed informed consents. This research was
Table I Correlation Between circHIPK2 Expression and Clinicopathological Characteristics in Ovarian Cancer

\begin{tabular}{|c|l|l|l|l|}
\hline \multirow{2}{*}{$\begin{array}{l}\text { Clinicopathologic } \\
\text { Parameters }\end{array}$} & Case & \multicolumn{2}{|c|}{$\begin{array}{c}\text { circHIPK2 } \\
\text { Expression }\end{array}$} & \multirow{2}{*}{ P value } \\
\cline { 2 - 4 } & & $\begin{array}{l}\text { Low } \\
\text { (n=23) }\end{array}$ & $\begin{array}{l}\text { High } \\
\text { (n=23) }\end{array}$ & \\
\hline $\begin{array}{l}\text { Age (years) } \\
\leq 50\end{array}$ & 18 & 7 & 11 & 0.2269 \\
$>50$ & 28 & 16 & 12 & \\
\hline $\begin{array}{c}\text { Tumor size } \\
\leq . \text { cm } \\
>4 \text { cm }\end{array}$ & 20 & 14 & 6 & $0.0173^{*}$ \\
\hline $\begin{array}{l}\text { Lymph node metastasis } \\
\text { Negative } \\
\text { Positive }\end{array}$ & 26 & 9 & 17 & \\
\hline $\begin{array}{l}\text { FIGO stage } \\
\text { I/II } \\
\text { III/IV }\end{array}$ & 30 & 16 & 4 & $0.0133^{*}$ \\
\hline
\end{tabular}

Notes: The median of circHIPK2 expression level was used as the cutoff. $* P<0.05$. ${ }^{a}$ Chi-square test and Fisher's exact test.

Abbreviation: FIGO, Federation Internationale of Gynecologie and Obstetrigue.

approved by the Ethics Committee of Cancer Hospital of China Medical University, Liaoning Cancer Hospital \& Institute. This study was conducted in accordance with the Declaration of Helsinki.

\section{Cells and Cell Culture}

Human ovarian surface epithelium cell line (IOSE80), ovarian serous cystadenocarcinoma cell line (SKOV3), and ovarian endometrioid adenocarcinoma cell line (A2780) were from the BeNa Culture Collection (BNCC; Beijing, China) and National Institutes of HealthDevelopmental Therapeutics Program (NCI-DTP; Frederick, MD, USA). IOSE80, SKOV3 and A2780 cells were cultured in RPMI-1640 (Hyclone, Logan, UT, USA) supplemented with $10 \%$ fetal bovine serum (FBS; Hyclone) and $2 \mathrm{mM}$ Glutamine (Sigma-Aldrich, St. Louis, MO, USA) at $37^{\circ} \mathrm{C}$. DDP-resistant SKOV3 and A2780 (SKOV3/DDP and A2780/DDP) cells were induced by exposure to stepwise increasing DDP concentrations until they were capable of growing in medium containing $20 \mu \mathrm{M}$ DDP.

\section{Cell Transfection}

Small interfering RNA (siRNA) sequences directed against circHIPK2 (si-circHIPK2\#1, \#2 and \#3) and 
siRNA negative control (si-NC) were self-designed and synthesized by Sangon Biotech (Shanghai, China). The mimic of miR-338-3p (miR-338-3p mimic) and inhibitor of miR-338-3p (anti-miR-338-3p) were synthesized by Sangon Biotech, as well as miR-NC mimic and antimiR-NC. CHTOP overexpression vector was constructed in pEGFP-N1 (pEGFP; Addgene, Watertown, MA, USA) by inserting the full-length cDNA sequence of CHTOP. The empty pEGFP vector was used as negative control of recombinant pEGFP-CHTOP. SKOV3/DDP and A2780/ DDP cells were re-seeded for $24 \mathrm{~h}$ prior to cell transfection. Reagent quantities of nucleotides and RNAifectin reagent (Abm, Richmond, BC, Canada) or DNAfection 2100 reagent $(\mathrm{Abm})$ were employed according to the corresponding transfection protocols. Short-hairpin RNA (shRNA) against circHIPK2 (sh-circHIPK2) and sh-NC were severally inserted into pSilencer 4.1-CMV puro vector (Invitrogen, Carlsbad, CA, USA). The stably transfected cells were then selected by $2.5 \mu \mathrm{g} / \mathrm{mL}$ puromycin (Sigma-Aldrich). For transient transfection, SKOV3/DDP and A2780/DDP cells were harvested after transfection for $24 \mathrm{~h}$ unless indicated otherwise. The sequences of oligonucleotides are presented in Table 2.

\section{Quantitative Reverse Transcription PCR (RT-qPCR) and Ribonuclease R (RNase R) Treatment}

Total RNA in tissues and cells was extracted with TRIpure reagent (BioTeke, Beijing, China) according to the manufacturer's instructions. Total RNA was used to synthesize cDNA with All-in-one MasterMix (Abm) and All-in-One miRNA First strand cDNA Synthesis Kit (GeneCopoeia, Rockville, MD, USA). The cDNAs were analyzed with BrightGreen $2 \times \mathrm{qPCR}$ MasterMix-ROX (Abm) and BrightGreen miRNA qPCR MasterMix-ROX (Abm) on 7500 Real-Time PCR System (Applied Biosystems, Foster City, CA, USA). The relative expression levels of circHIPK2, miR-338-3p and CHTOP mRNA were detected and normalized to U6 small nuclear RNA (RNU6) or glyceraldehyde-3-phosphate dehydrogenase (GAPDH) using $2^{-\Delta \Delta \mathrm{Ct}}$ method. The primers are presented in Table 2.

For RNase R treatment, total RNA ( $2 \mu \mathrm{g}$ ) was incubated with or without $3 \mathrm{U}$ RNase R (Solarbio, Beijing,
Table 2 The Primers, Oligonucleotides and Probes Sequences Used in This Study

\begin{tabular}{|c|c|}
\hline Name & Sequence \\
\hline \multirow[t]{2}{*}{$\begin{array}{l}\text { circHIPK2 } \\
\text { (145 nt) }\end{array}$} & $\begin{array}{l}\text { 5'-TGCTCCACCTACTTGCAGTC-3' } \\
\text { (forward) }\end{array}$ \\
\hline & 5'-GTACCCAGTCATGTCCCAGT-3' (reverse) \\
\hline \multirow[t]{2}{*}{$\begin{array}{l}\text { linear HIPK2 } \\
\text { (I } 43 \mathrm{nt})\end{array}$} & $\begin{array}{l}\text { 5'-AGCCAGAGCAAGAACATCCC-3' } \\
\text { (forward) }\end{array}$ \\
\hline & 5'-CTTGCTGAGGTGACCACGAT-3' (reverse) \\
\hline \multirow{2}{*}{$\begin{array}{l}\mathrm{miR}-338-3 p \\
(102 \mathrm{nt})\end{array}$} & 5'-ATATCCTGGTGCTGAGTG -3' (forward) \\
\hline & 5'-GAACATGTCTGCGTATCTC-3' (reverse) \\
\hline \multirow[t]{2}{*}{ CHTOP (I22 nt) } & $\begin{array}{l}\text { 5'-GCTTCGATGCAGCAACAACA-3' } \\
\text { (forward) }\end{array}$ \\
\hline & 5'-AGGCGCTGCTTTAAGCTCTG-3' (reverse) \\
\hline \multirow[t]{2}{*}{ GAPDH (I22 nt) } & $\begin{array}{l}\text { 5'-GGCCTCCAAGGAGTAAGACC-3' } \\
\text { (forward) }\end{array}$ \\
\hline & $\begin{array}{l}\text { 5'-AGGGGAGATTCAGTGTGGTG-3' } \\
\text { (reverse) }\end{array}$ \\
\hline \multirow[t]{2}{*}{ RNU6 (90 nt) } & 5'-CTCGCTTCGGCAGCACAT-3' (forward) \\
\hline & 5'-AACGCTTCACGAATTTGCGT-3' (reverse) \\
\hline si-circHIPK2\#I & 5'-GAGGCCAUACCUGGGCAAGCTT-3' \\
\hline si-circHIPK2\#2 & 5'-AUACCUGUAAUAUCUGGACTT-3' \\
\hline si-circHIPK2\#3 & 5'-CAUGUGAGGCCAUACCUGGTT-3' \\
\hline si-NC & 5'-TGTCTGGTTGACATCATGCTTAATG-3' \\
\hline \multirow[t]{2}{*}{ sh-circHIPK2 } & 5'-GUCCAGAUAUUACAGGUAUTT-3' (sense) \\
\hline & $\begin{array}{l}\text { 5'-TTAUACCUGUAAUAUCUGGAC-3' (anti- } \\
\text { sense) }\end{array}$ \\
\hline \multirow[t]{2}{*}{ sh-NC } & $\begin{array}{l}\text { 5'-TGTCTGGTTGACATCATGCTTAATG-3' } \\
\text { (sense) }\end{array}$ \\
\hline & $\begin{array}{l}\text { 5'-CATTAAGCATGATGTCAACCAGACA-3' } \\
\text { (antisense) }\end{array}$ \\
\hline $\begin{array}{l}\text { miR-338-3p } \\
\text { mimic }\end{array}$ & 5'-UCCAGCAUCAGUGAUUUUGUUG-3' \\
\hline miR-NC mimic & 5'-UCUCCGAACGUGUCACGU-3' \\
\hline Anti-miR-338-3p & 5'-CAACAAAAUCACUGAUGCUGGA-3' \\
\hline Anti-miR-NC & 5'-CAGUACUUUUGUGUAGUACAA-3' \\
\hline Bio-circHIPK2 & biotin-ACCAGAAAACAUCAUGCUGGU \\
\hline Bio-CHTOP & biotin-AGAAGAAAUCUGAUUGAUGCUGGA \\
\hline
\end{tabular}


China) for $30 \mathrm{~min}$ at $37^{\circ} \mathrm{C}$. The expression levels of circHIPK2 and linear HIPK2 were detected by RT-qPCR. Agarose gel electrophoresis showed the RT-qPCR products in both RNase $\mathrm{R}$ group and mock group.

\section{Cell Counting Kit (CCK)-8 Assay}

Cell viability was measured by CCK-8 (MCE, Shanghai, China). For determination of $50 \%$ inhibitory concentration (IC50) of DDP, different groups of SKOV3/DDP and A2780/DDP cells (8000 cells/well) were seeded in 96well plate for overnight with five replicates. These cells were treated with DDP at different concentrations (0-120 $\mu \mathrm{M})$ for $24 \mathrm{~h}$. DDP was diluted in phosphate buffer saline (PBS; $\mathrm{pH}$ 7.4). Then, $10 \mu \mathrm{L}$ CCK-8 solution was added to each well for another $2 \mathrm{~h}$-incubation. After mixing on orbital shaker for $5 \mathrm{~min}$, the absorbance at $450 \mathrm{~nm}$ was read using a microplate reader. Cell viability (\%) was normalized to control group $(0 \mu \mathrm{M})$, and IC50 of DDP was calculated according to cell viability curve.

For evaluation of cell proliferation, SKOV3/DDP and A2780/DDP cells (5000 cells/well) were plated in 96-well plates with five replicates, and transfected with corresponding nucleotides. CCK-8 solution was added at indicated time points $(0,24,48$, and $72 \mathrm{~h})$, and optical density (OD) value at $450 \mathrm{~nm}$ was measured; cell proliferation curve was drawn according to OD values.

\section{Flow Cytometry (FCM)}

Cell Cycle and Apoptosis Analysis Kit (Yeasen, Shanghai, China) was employed to measure cell distribution in phases. Transfected SKOV3/DDP and A2780/DDP cells $\left(5 \times 10^{5}\right)$ were collected and fixed in $70 \%$ ethanol for overnight at $4^{\circ} \mathrm{C}$. Next, $10 \mu \mathrm{L}$ propidium iodide (PI) solution and $10 \mu \mathrm{L}$ RNase A were mixed in the Staining Solution, where fixed cells were incubated for $30 \mathrm{~min}$ at $37^{\circ} \mathrm{C}$. The stained cells were analyzed on Attune ${ }^{\mathrm{TM}} \mathrm{NxT}$ Acoustic Focusing Cytometer (Invitrogen).

Apoptosis rate of SKOV3/DDP and A2780/DDP cells was examined by Annexin V-fluorescein isothiocyanate (FITC)/PI Apoptosis Detection Kit (Yeasen). After transfection, a set of $5 \times 10^{5}$ cells were collected and resuspended in $1 \times$ Binding Buffer. Then, $5 \mu \mathrm{L}$ Annexin V-FITC and $10 \mu \mathrm{L}$ PI Staining Solution were added for cell incubation for $20 \mathrm{~min}$ at $25^{\circ} \mathrm{C}$ free-from light. The stained cells were analyzed on FCM, and apoptosis rate (\%) was calculated on Cell Quest software (BD Biosciences, San Jose, CA, USA).

\section{Transwell Assays}

For transwell migration assay, approximately $1 \times 10^{5}$ cells (SKOV3/DDP and A2780/DDP) after different transfections were re-suspended in serum-free RPMI-1640 (Hyclone) and plated in the upper place of transwell chamber $(8.0 \mu \mathrm{m}$ pore size, Corning, Kennebunk, ME, USA). For transwell invasion assay, transfected SKOV3/ DDP and A2780/DDP cells were subjected to similar procedures in transwell chamber supplemented with Matrigel filter (BD Biosciences). The lower place of transwell chambers was filled with RPMI-1640 containing 10\% FBS (Hyclone) to avoid touching the bottom of chambers. After incubation for $48 \mathrm{~h}$, the transferred cells were fixed with $4 \%$ paraformaldehyde and stained with $0.5 \%$ crystal violet (Yeasen). The stained cells were photographed under microscope at $100 \times$ amplification, and the number of migrated/invaded cells per field was counted according to five random fields.

\section{Western Blotting}

Proteins were extracted from tissues and cells utilizing Lysis Buffer (Yeasen) containing a protease inhibitor cocktail (Beyotime). An equal amount of protein lysates (30 $\mu \mathrm{g}$ ) was separated on $10 \%$ or $12 \%$ SDS-polyacrylamide gel electrophoresis, and then transferred onto polyvinylidene difluoride membranes. The protein-carrying membranes were incubated with primary antibodies for overnight at $4{ }^{\circ} \mathrm{C}$, and with secondary antibodies for 1.5 $\mathrm{h}$ at $25^{\circ} \mathrm{C}$. GAPDH was chosen as internal control, and gray density of Western blots was analyzed on Image J software (NIH, Bethesda, MD, USA). Relative protein expression levels of B cell lymphoma (Bcl)-2, Bcl-2-associated $\mathrm{X}$ protein (Bax), matrix metalloproteinase 2 (MMP2) and MMP9 were calculated with normalization to GAPDH. The antibodies are summarized in Table 3.

\section{Xenograft Tumor Models}

This animal study was approved by the Animal Ethics Committee of Cancer Hospital of China Medical University, Liaoning Cancer Hospital \& Institute, and BALB/c-nude mice (female, 4-5 weeks of age) were purchased from Shanghai Experimental Animal Center (Shanghai, China). Stably transfected A2780/DDP cells $\left(1 \times 10^{7}\right.$ cells in $0.2 \mathrm{~mL}$ PBS $)$ were subcutaneously injected into the posterior flank of the nude mice, and tumors were generated after cell inoculation for 7 days. Xenografts were randomly divided into DDP-treated group and PBS-treated 
Table 3 The Antibodies Used in This Study

\begin{tabular}{|l|l|l|l|}
\hline Name & Cat. No. & $\begin{array}{l}\text { Dilution } \\
\text { Ratio }\end{array}$ & Source \\
\hline CHTOP & ab22286I & I:5000 & Abcam \\
\hline Bcl-2 & ab32124 & I:1000 & Abcam \\
\hline Bcl-2 & abl96495 & I:2000 & Abcam \\
\hline Bax & ab32503 & I:10,000 & Abcam \\
\hline Bax & ab319I & I:500 & Abcam \\
\hline MMP2 & ab86607 & I:500 & Abcam \\
\hline MMP9 & abl37867 & I:1000 & Abcam \\
\hline MMP9 & ab38898 & I:1000 & Abcam \\
\hline GAPDH & abl81602 & I:10,000 & Abcam \\
\hline $\begin{array}{l}\text { Goat anti-Rabbit IgG } \\
\text { (HRP) }\end{array}$ & ab2057I8 & I:50,000 & Abcam \\
\hline $\begin{array}{l}\text { Goat anti-mouse IgG } \\
\text { (HRP) }\end{array}$ & ab6789 & I:10,000 & Abcam \\
\hline
\end{tabular}

group ( $\mathrm{n}=6)$. Then, cisplatin $(5 \mathrm{mg} / \mathrm{kg}$ ) or $0.2 \mathrm{~mL}$ PBS was intraperitoneally injected in nude mice every 3.5 days for another 28 days. Tumor diameter was monitored every 7 days using calipers, and tumor volume was calculated using the formula: $0.5 \times\left(\right.$ length $\times$ width $\left.^{2}\right)$. The mice were euthanized, and the tumors were dissected, weighted and photographed. The tumor tissues were collected and restored in $-80^{\circ} \mathrm{C}$ for further RNA/protein isolation. All animal experiments were performed following the Guide for the Care and Use of Laboratory Animals (GB/T 35892-2018; Standardization Administration of the People Republic of China).

\section{Dual-Luciferase Reporter Assay}

The predicted miR-338-3p-binding sites in human circHIPK2 and 3'-untranslated region of CHTOP (CHTOP 3'UTR) were directly mutated, and PCR method was used to amplify the wild type (WT) and mutant type (MUT) of circHIPK2 and CHTOP 3'UTR. CircHIPK2 WT/MUT and CHTOP 3'UTR WT/MUT were severally inserted into pGL4 reporter vectors (Promega, Madison, WI, USA). SKOV3/DDP and A2780/DDP cells in 96-well plate were co-transfected with reporter vectors and either miR-338-3p mimic or miR-NC mimic for $48 \mathrm{~h}$. The luciferase activity of cells was measured by the DualLuciferase Reporter Assay Kit (Promega) on GloMax
Discover Microplate Reader (Promega). Renilla luciferase served as endogenous control.

\section{RNA Pull-Down Assay}

The sequences of miR-338-3p-binding sites in circHIPK2 and CHTOP 3'UTR were severally subjected to biotinylation using T7 High Yield Transcription Kit (Thermo Fisher Scientific, Waltham, MA, USA). SKOV3/DDP and A2780/DDP cells were transfected with biotinylated circHIPK2 (bio-circHIPK2) or biotinylated CHTOP 3'UTR (biotin-CHTOP), and cell extract was obtained in RIP lysis buffer. Cell extract was subjected to Dynabeads MyOne Streptavidin beads (Invitrogen) for $12 \mathrm{~h}$ at $4^{\circ} \mathrm{C}$. After eluting, RNA complexes bound to beads were captured, and then extracted by TRIpure reagent (BioTeke). The cell extract before beads incubation served as the Input, and miR-338-3p enrichment in Input and contents mediated by biotin-HIPK2 and biotin-CHTOP was subsequently analyzed by RT-qPCR.

\section{Statistical Analysis}

Statistical analysis was determined by GraphPad Prism 5.0 software (GraphPad, San Diego, CA, USA) according to mean ( \pm standard deviation) from at least three independent experiments. Student's $t$-test and analysis of variance (ANOVA) analysis were performed for comparisons. Tukey's post-hoc analysis was employed after ANOVA. A comparison with $P<0.05$ was deemed as statistically significant.

\section{Results}

\section{CircHIPK2 Was Upregulated in OvCa Patients' Tumors in Association with DDP Resistance}

We established two sub-lines of DDP-resistant OvCa cells depending on SKOV3 and A2780 cell lines, and IC50 of DDP values were, respectively, increased in SKOV3/DDP, and A2780/DDP cells than parental cells (Figure 1A and B). Sanger sequencing identified the back splice junction of circHIPK2 (Figure 1C). Comparing to corresponding control tissues, expression of circHIPK2 was upregulated not only in OvCa patients' tumors but also in DDPresistant OvCa tumors (Figure 1D and E). Moreover, circHIPK2 high expression was correlated with clinicopathological characteristics, such as tumor size, lymph node metastasis, and advanced FIGO stage (Table 1). Its expression level was overall higher in human $\mathrm{OvCa}$ cell 


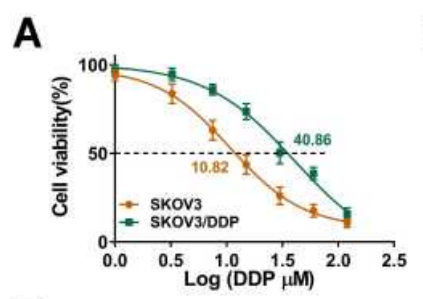

E

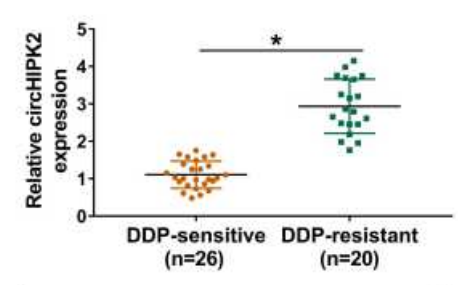

\section{I}

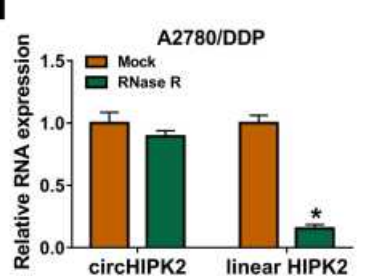

B

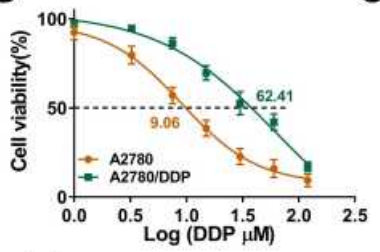

F
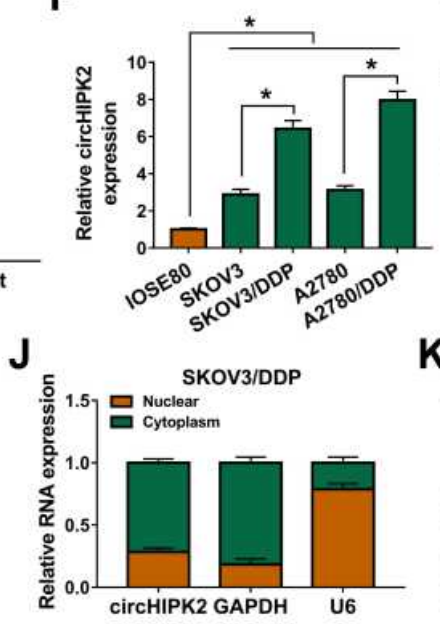

C

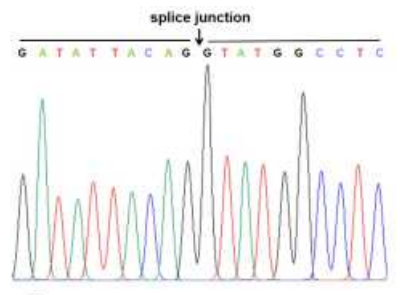

G

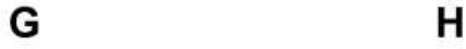

H

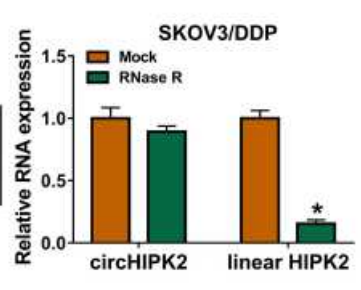

D

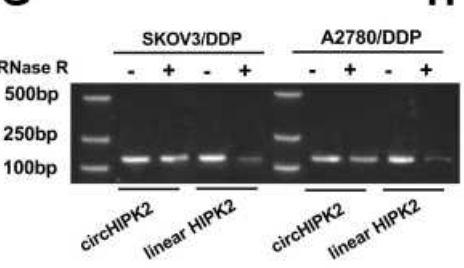

K

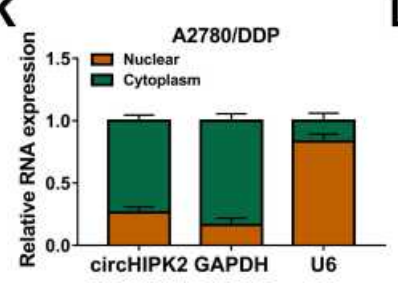

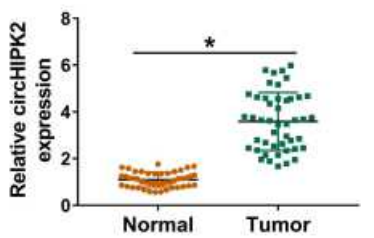

L

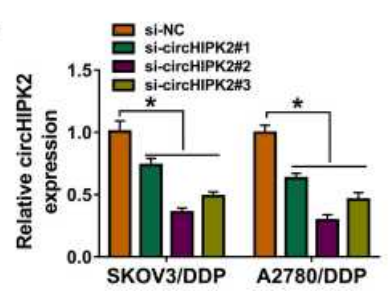

Figure I Expression of circHIPK2 in OvCa patients and DDP-resistant cells. (A and B) CCK-8 assay measured cell viability (\%) of SKOV3, SKOV3/DDP, A2780, and A2780/ DDP cells severally treated with I-I20 $\mu$ M DDP, and IC50 value of DDP was calculated. (C) Sanger sequencing showed the splice junction of circHIPK2. (D and E) RT-qPCR detected circHIPK2 expression in OvCa tumor tissues (Tumor; $n=46$ ) and paired normal tissues (Normal; $n=46$ ), and DDP-resistant tumor tissues ( $\mathrm{n}=20$ ) and DDP-sensitive tumor tissues $(n=26)$. (F) RT-qPCR detected circHIPK2 expression in IOSE80, SKOV3, SKOV3/DDP, A2780, and A2780/DDP cells. (G) Agarose gel electrophoresis and (H and I) RT-qPCR showed RNA expression of circHIPK2 and linear HIPK2 in RNase R-treated and RNase R-untreated total RNAs extracted from SKOV3/DDP and A2780/ DDP cells. (J and K) RT-qPCR detected RNA expression of circHIPK2, GAPDH and U6 in the cytoplasm fraction and nuclear fraction of SKOV3/DDP and A2780/DDP cells. (L) RT-qPCR examined circHIPK2 expression in SKOV3/DDP and A2780/DDP cells severally transfected with si-circHIPK2\#I, \#2 and \#3, compared to si-NC transfection. $* P<0.05$. Data analysis was performed using unpaired t-test and one-way or two-way ANOVA followed with Tukey's post-hoc analysis.

lines (SKOV3 and A2780), and even higher in DDPresistant OvCa cells (SKOV3/DDP, and A2780/DDP) (Figure 1F); notably, circHIPK2 expression was little affected by RNase R, whereas linear HIPK2 expression was dramatically reduced with RNase $\mathrm{R}$ digestion. (Figure 1G-I). Besides, circHIPK2 expression was predominantly discovered in cytoplasm fraction of SKOV3/DDP and A2780/DDP cells (Figure 1J and K). These data demonstrated that circHIPK2 was upregulated in OvCa and was associated with DDP resistance.

\section{Knockdown of circHIPK2 Reversed DDP Resistance and Restrained Malignant Behaviors of DDP-Resistant OvCa Cells}

Thus, the high expression of circHIPK2 was forcedly silenced using special siRNAs transfection, and sicircHIPK2\#2 caused the optimal knockdown efficiency in both SKOV3/DDP and A2780/DDP cells (Figure 1L). CircHIPK2 silencing experiments were performed using
si-circHIPK2\#2. First of all, IC50 values of DDP were significantly decreased in circHIPK2-silenced SKOV3/ DDP and A2780/DDP cells (Figure 2A and B).Cell proliferation of SKOV3/DDP and A2780/DDP cells analyzed by CCK-8 assay was inhibited by si-circHIPK2\#2 transfection, as indicated by lowered OD values at $48 \mathrm{~h}$ and 72 $\mathrm{h}$ (Figure 2C and D). On the contrary, FCM method showed that apoptosis rate of circHIPK2-interferred SKOV3/DDP and A2780/DDP cells was greatly promoted (Figure 2E); similarly, cells arrested in G0/G1 phase were increased, and cells entranced in $\mathrm{S}$ phase were decreased in SKOV3/DDP and A2780/DDP cells transfected with sicircHIPK2\#2 (Figure 2F and G). Transwell migrated cells and invaded cells were both diminished in the presence of si-circHIPK2\#2 than si-NC (Figure $2 \mathrm{H}$ and I). Molecularly, apoptosis-related proteins and migrationassociated proteins were abnormally expressed with circHIPK2 downregulation, as evidenced by the elevation of Bax, and the reduction of Bcl-2, MMP2 and MMP9 (Figure $2 \mathrm{~J}$ and $\mathrm{K}$ ). These results indicated that blocking 
A

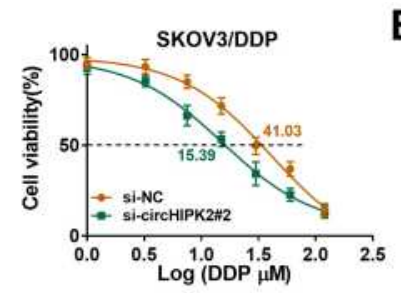

E

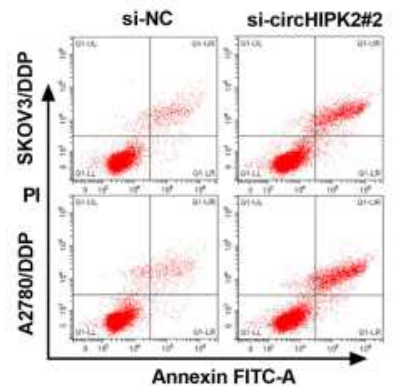

G
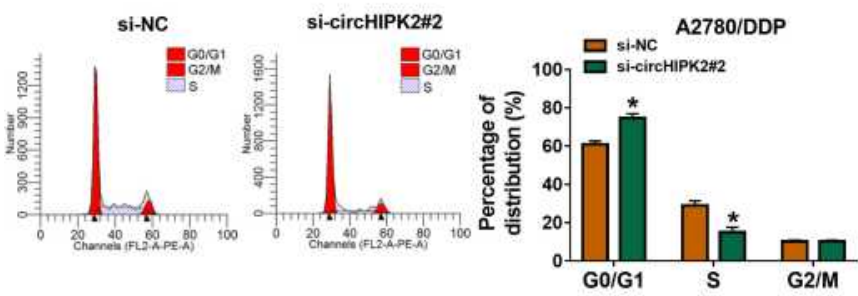

I
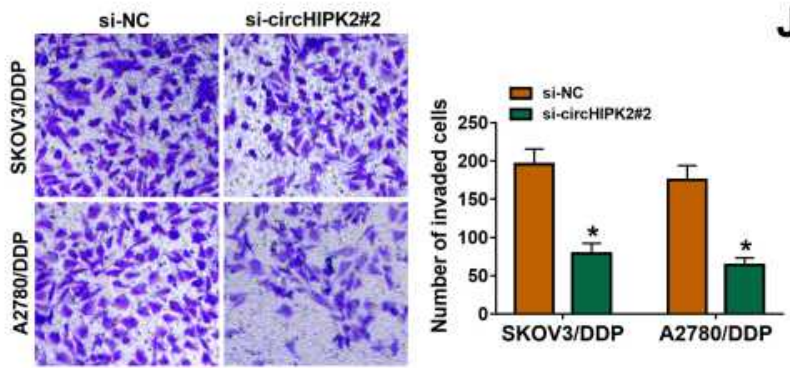

K
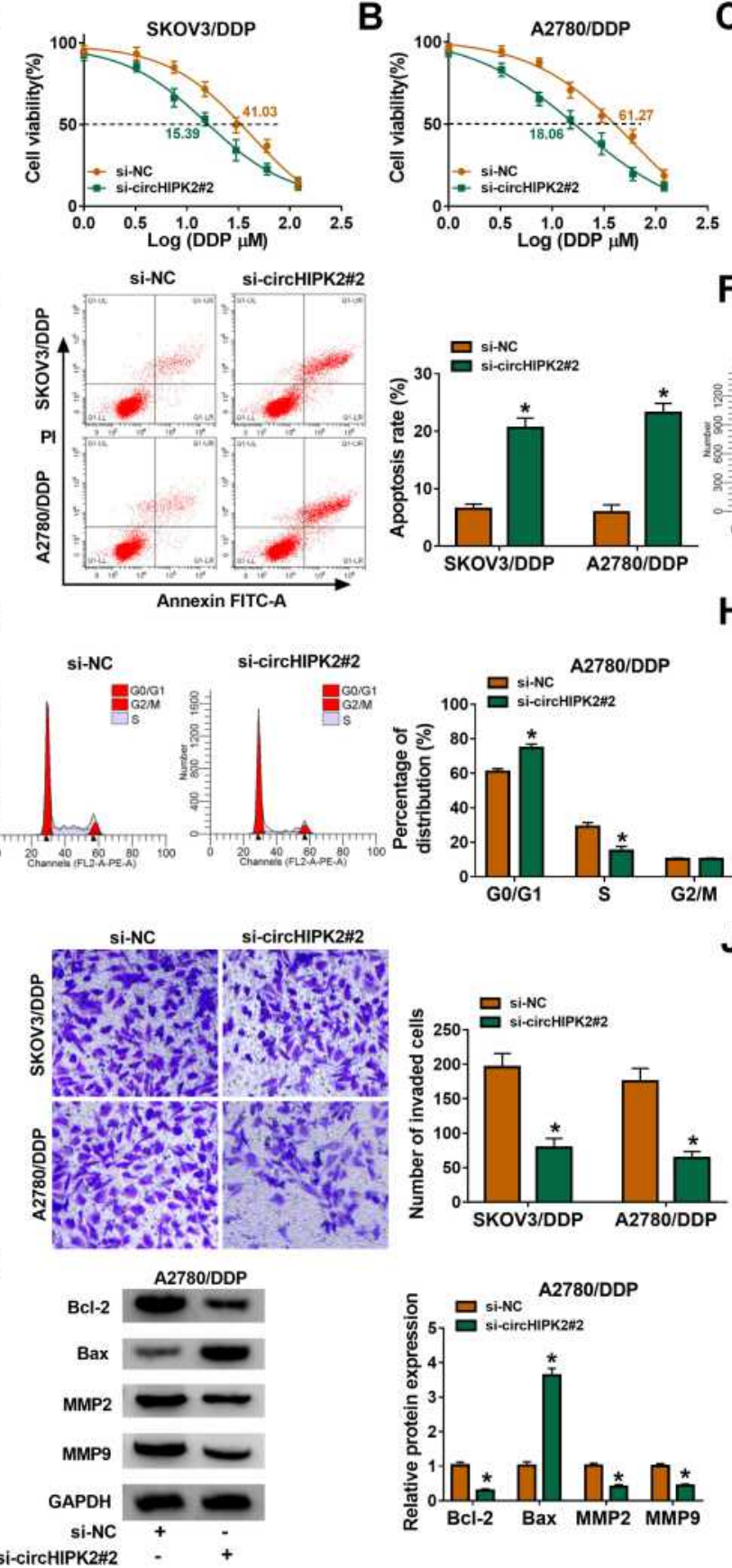
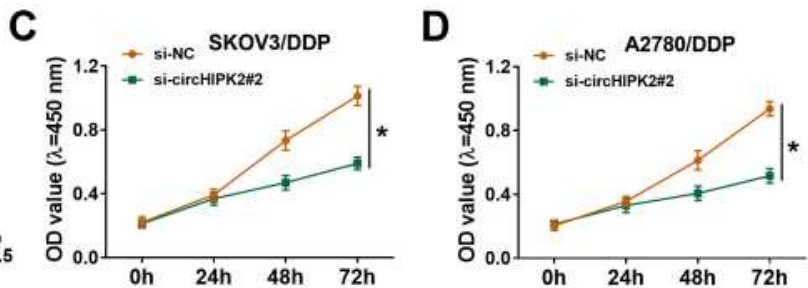

F
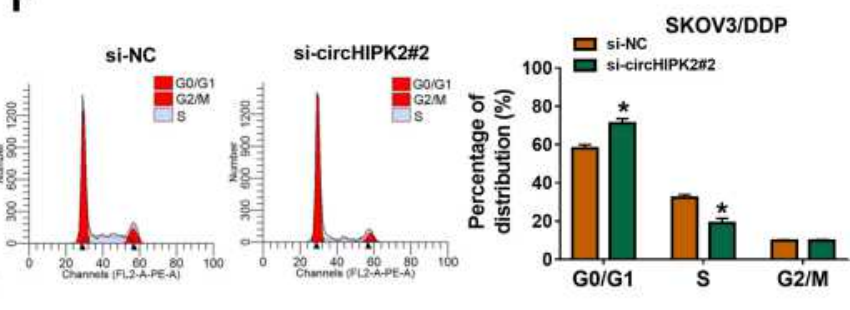

H
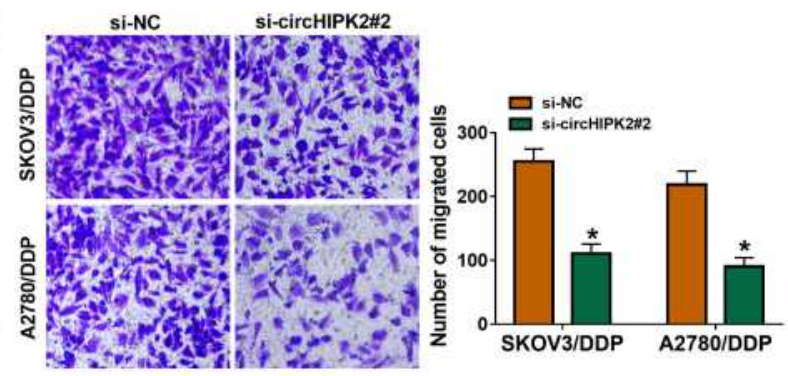

J
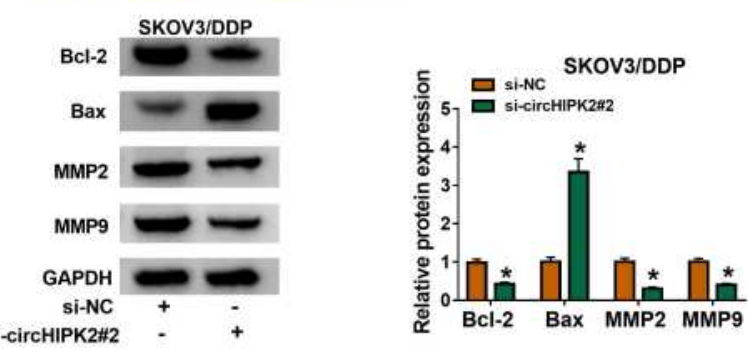

Figure 2 Role of circHIPK2 knockdown in DDP resistance and malignant behaviors of DDP-resistant OvCa cells in vitro. SKOV3/DDP and A2780/DDP cells were transfected with si-NC or si-circHIPK2\#2. (A and B) CCK-8 assay measured IC50 of DDP by monitoring cell viability (\%) after treatment of I-I20 $\mu$ M DDP. (C and D) CCK-8 assay showed optical density (OD) values at $450 \mathrm{~nm}$ after transfection for 0-72 h. After transfection for $24 \mathrm{~h},(\mathbf{E}-\mathbf{G})$ FCM method examined apoptosis rate (\%) and percentages of cell distribution in G0/GI, S and G2/M phases, ( $\mathbf{H}$ and $\mathbf{I})$ transwell assay evaluated numbers of migrated cells and invaded cells, and $(\mathbf{J}$ and $\mathbf{K})$ Western blotting detected protein expression of Bcl-2, Bax, MMP2, and MMP9, normalized to GAPDH. ${ }^{*} P<0.05$. Data analysis was performed using two-way ANOVA followed with Tukey's post-hoc analysis.

circHIPK2 could suppress DDP resistance, proliferation, cell cycle progression, migration and invasion of DDPresistant OvCa cells in vitro but promoted apoptosis.

Xenograft tumor models were employed using A2780/ DDP cells, and xenograft tumor volume and weight were restrained in sh-circHIPK2-transfected groups with DDP treatment or PBS treatment (Figure 3A-C). Furthermore, circHIPK2 expression in xenograft tumors' tissues was silenced in sh-circHIPK2 groups than corresponding sh$\mathrm{NC}$ groups (Figure 3D). Allied with that was the inhibition 

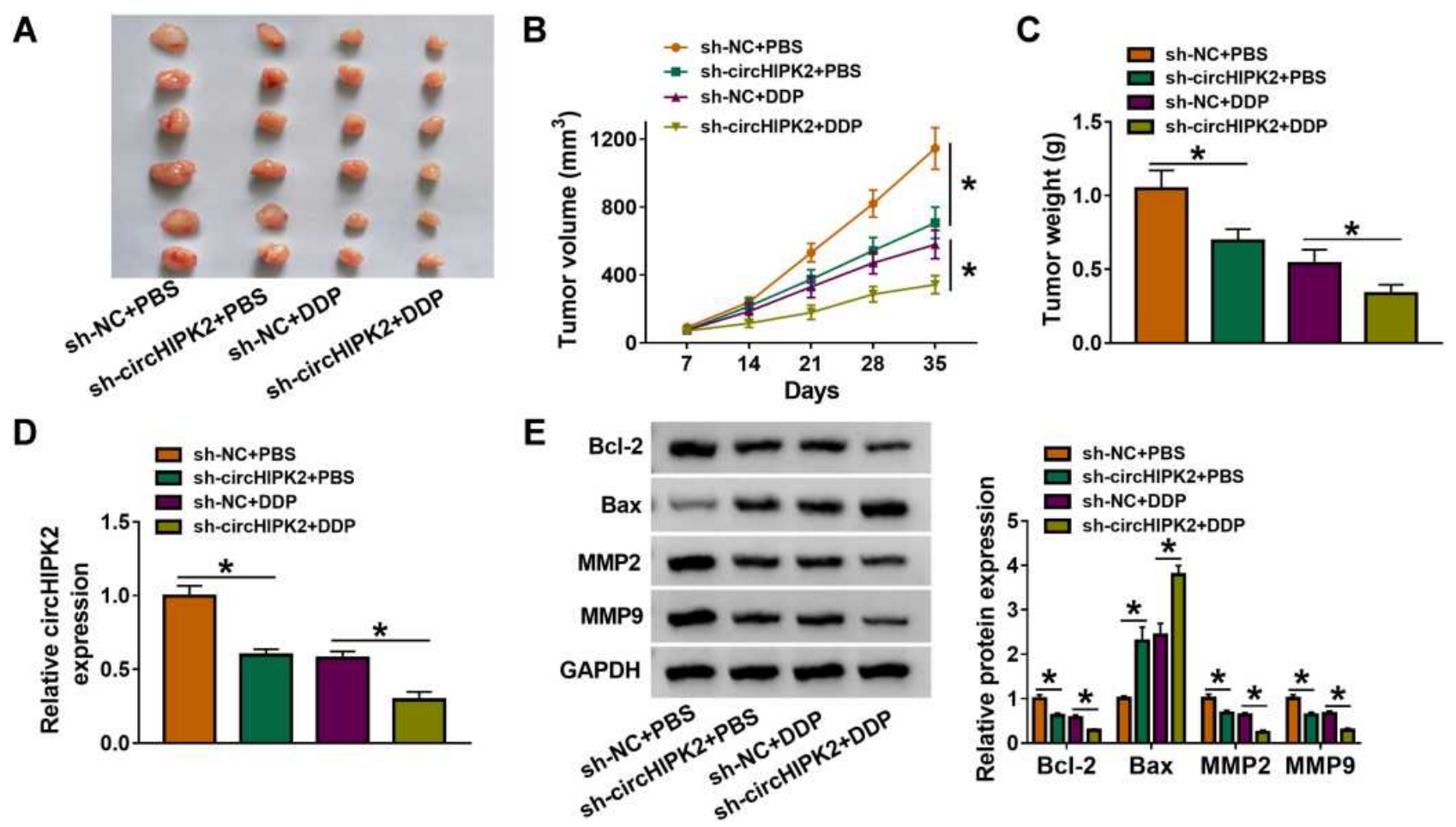

Figure 3 Role of circHIPK2 knockdown in DDP resistance and tumorigenicity of DDP-resistant OvCa cells in vitro. A2780/DDP cells stably transfected with sh-NC or shcircHIPK2 were inoculated in nude mice, followed with DDP or PBS treatment. (A) Xenograft tumors in each group ( $\mathrm{n}=6$ ) were presented. (B) Tumor volume was monitored every 7 days. (C) Tumor weight was examined after cell inoculation for 35 days. (D) RT-qPCR detected circHIPK2 expression and (E) Western blotting detected protein expression of $\mathrm{Bcl}-2$, Bax, MMP2, and MMP9 in xenograft tumor tissues. $* P<0.05$. Data analysis was performed using one-way or two-way ANOVA followed with Tukey's post-hoc analysis.

of Bcl-2, MMP2 and MMP9, and the enhancement of Bax in the sh-circHIPK2 groups (Figure 3E). These results suggested a suppressive role of circHIPK2 interference in the tumorigenicity of DDP-resistant cells in vivo.

\section{CircHIPK2 Functioned as a Sponge for miR-338-3p in DDP-Resistant OvCa Cells}

Next, miRNA binding site prediction databases showed 5 potential target miRNAs for circHIPK2 (Figure 4A). Among these miRNAs, miR-338-3p, miR-584-5p and miR-889-3p expression could be significantly upregulated in si-circHIPK2\#2-transfected SKOV3/DDP and A2780/ DDP cells (Figure 4B and C), and miR-338-3p was the most upregulated one. Thus, the intact miR-338-binding sites in circHIPK2 were directly mutated (Figure 4D), and dual-luciferase reporter assay revealed that luciferase activity of reporter vector carrying circHIPK2 MUT failed to response to miR-338-3p overexpression in SKOV3/ DDP and A2780/DDP cells (Figure 4E and F). Meanwhile, miR-338-3p was abundantly enriched in biocircHIPK2-mediated pull-down contents than bio-NC (Figure 4G and $\mathrm{H}$ ). Expression of miR-338-3p was lower in human OvCa tumors and cells (Figure 4I and K), and even lower miR-338-3p level was discovered in DDPresistant human OvCa tumors and cells (Figure 4J and $\mathrm{K})$. These outcomes depicted that miR-338-3p was targeted by circHIPK2 and was downregulated in DDPresistant OvCa.

\section{Blocking miR-338-3p Could Abrogate Role of circHIPK2 Knockdown in DDP-Resistant OvCa Cells in vitro}

Rescue experiments showed that anti-miR-338-3p administration could weaken circHIPK2 knockdown-mediated upregulation of miR-338-3p in SKOV3/DDP and A2780/ DDP cells (Figure 5A). IC50 values of SKOV3/DDP and A2780/DDP cells were lowered by blocking circHIPK2, and this inhibition was counteracted by additionally blocking miR-338-3p via its inhibitor transfection (Figure 5B and C). The suppressive effect of circHIPK2 knockdown on proliferation of SKOV3/DDP and A2780/DDP cells and promoting effect on the apoptosis rate were both partially reversed by co-transfecting anti-miR-338-3p (Figure 5D-F). Cell arrest in G0/G1 phase mediated by si-circHIPK2\#2 
A

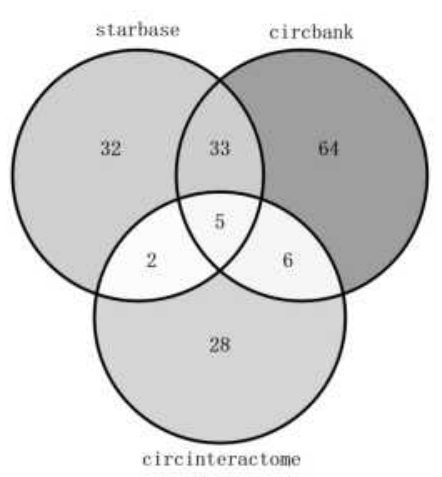

D

circHIPK2 WT 5' accaGAAAACA--UCAUGCUGGu 3' miR-338-3p 3' guugUUUUAGUGACUACGACCu 5' circHIPK2 MUT 5' accaGGCCAUG-CCGCAUGAUu 3'
$\mathbf{F}$

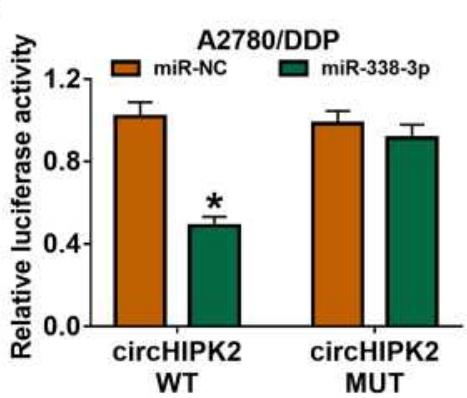

I

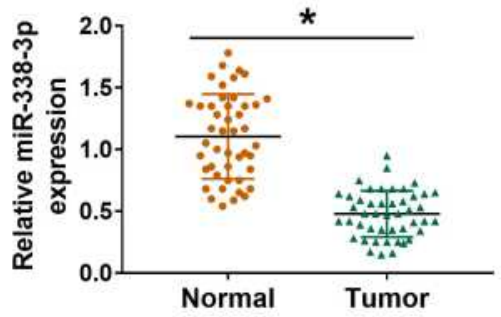

B

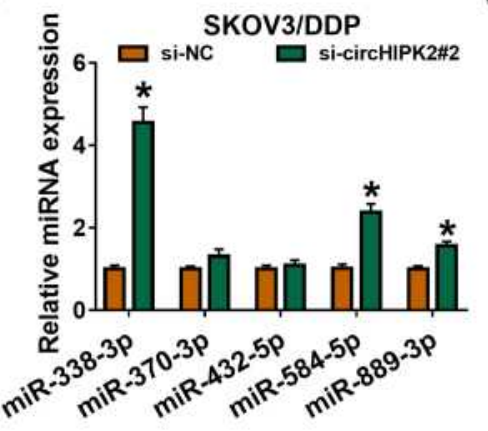

C

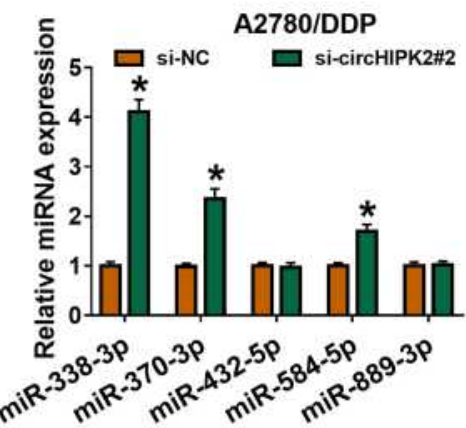

E

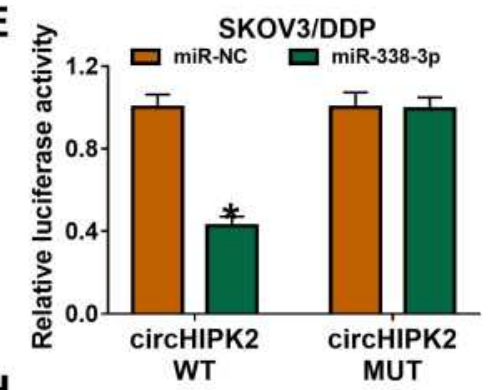

G

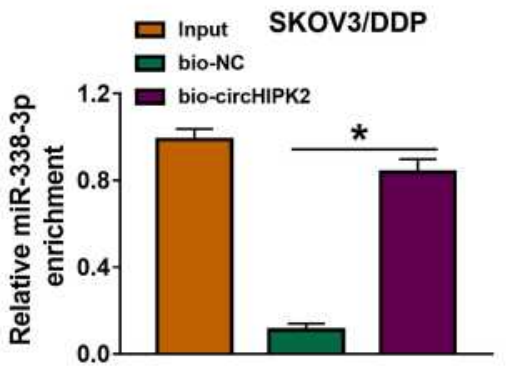

H

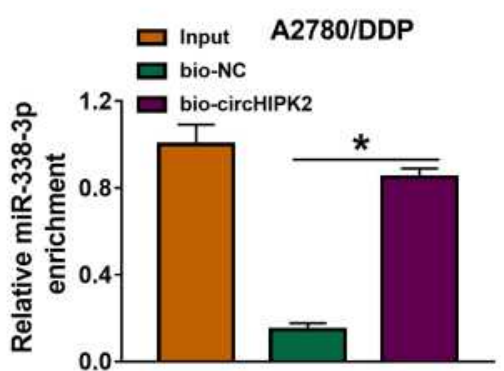

$J$

K

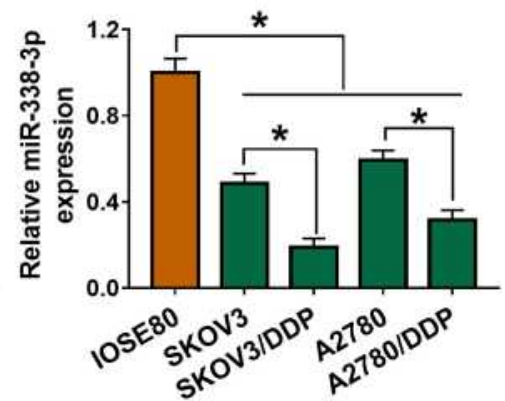

Figure 4 Identification of miR-338-3p as target of circHIPK2 in DDP-resistant OvCa cells. (A) Starbase, circinteractome and circbank databases together predicted miRNA binding sites in circHIPK2. (B and C) RT-qPCR detected expression of 5 miRNAs in SKOV3/DDP and A2780/DDP cells transfected with si-NC or si-circHIPK2\#2. (D) CircHIPK2 MUT was constructed by mutating the predicted binding sites of miR-338-3p in circHIPK2 WT. (E and F) Dual-luciferase reporter assay measured luciferase activity of reporter vectors carrying circHIPK2 WT or MUT in SKOV3/DDP and A2780/DDP cells co-transfected with miR-338-3p mimic (miR-338) or miR-NC mimic (miRNC). (G and H) RNA pull-down assay assessed miR-338-3p enrichment by bio-circHIPK2 or bio-NC in SKOV3/DDP and A2780/DDP cells. (I-K) RT-qPCR detected miR338-3p expression in (I) OvCa tumor tissues and normal tissues, (J) DDP-sensitive tumor tissues and DDP-resistant tumor tissues, as well as (K) cells (IOSE80, SKOV3, SKOV3/DDP, A2780, and A2780/DDP). $* P<0.05$. Data analysis was performed using unpaired $t$-test and one-way or two-way ANOVA followed with Tukey's post-hoc analysis.

transfection was rescued with the introduction of anti-miR -338-3p (Figure 5G and H). Numbers of transwell migrated cells and invaded cells were lower in circHIPK2-depleted SKOV3/DDP and A2780/DDP cells, which were improved with simultaneous depletion of miR-338-3p (Figure 5I and
J). Silencing circHIPK2 enhanced Bax expression, and depressed Bcl-2, MMP2 and MMP9 expression in SKOV3/DDP and A2780/DDP cells, and these protein expression levels were overall attenuated by simultaneously silencing miR-338-3p (Figure $5 \mathrm{~K}$ and L). These results 

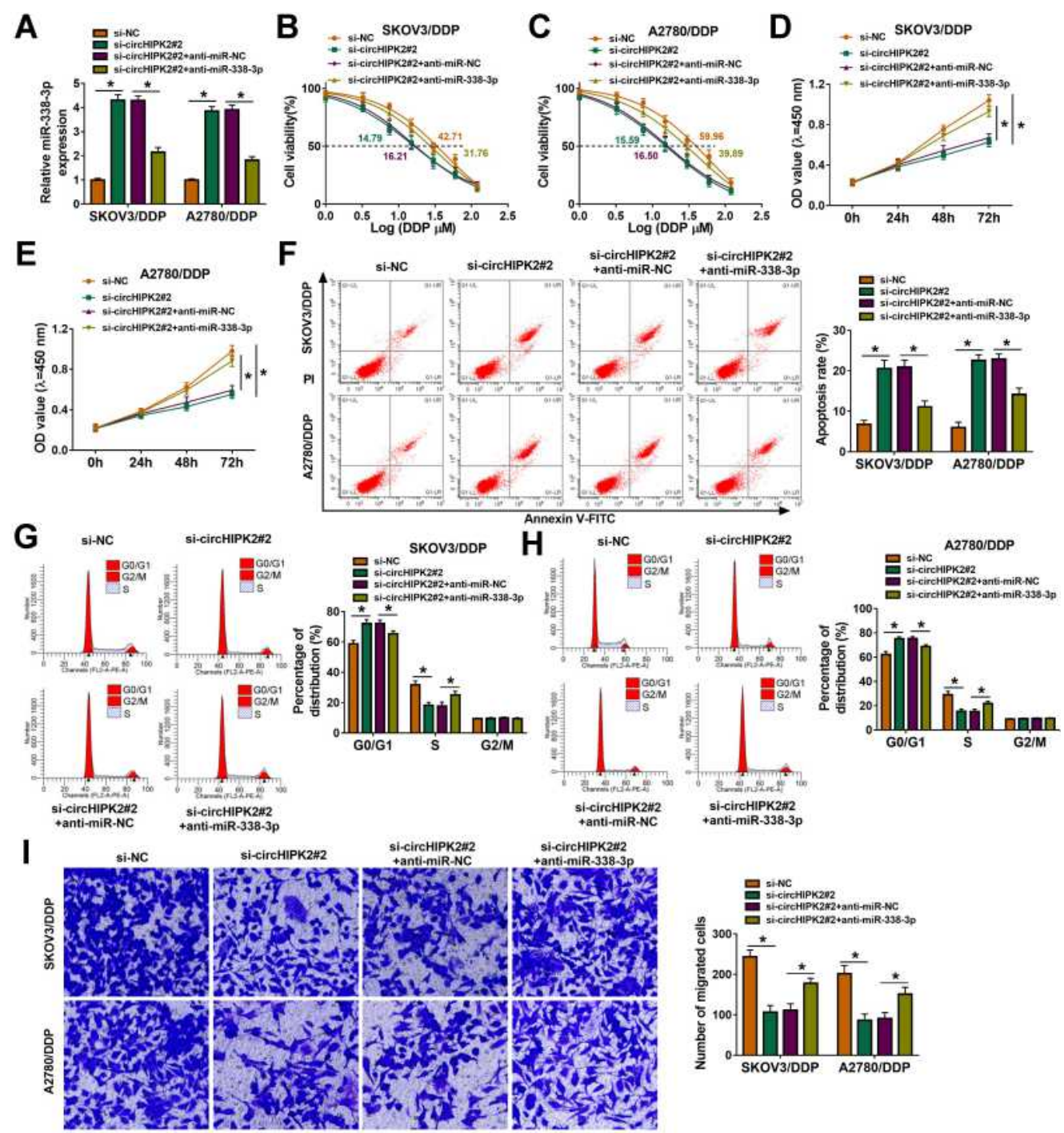

\section{$\mathbf{J}$}
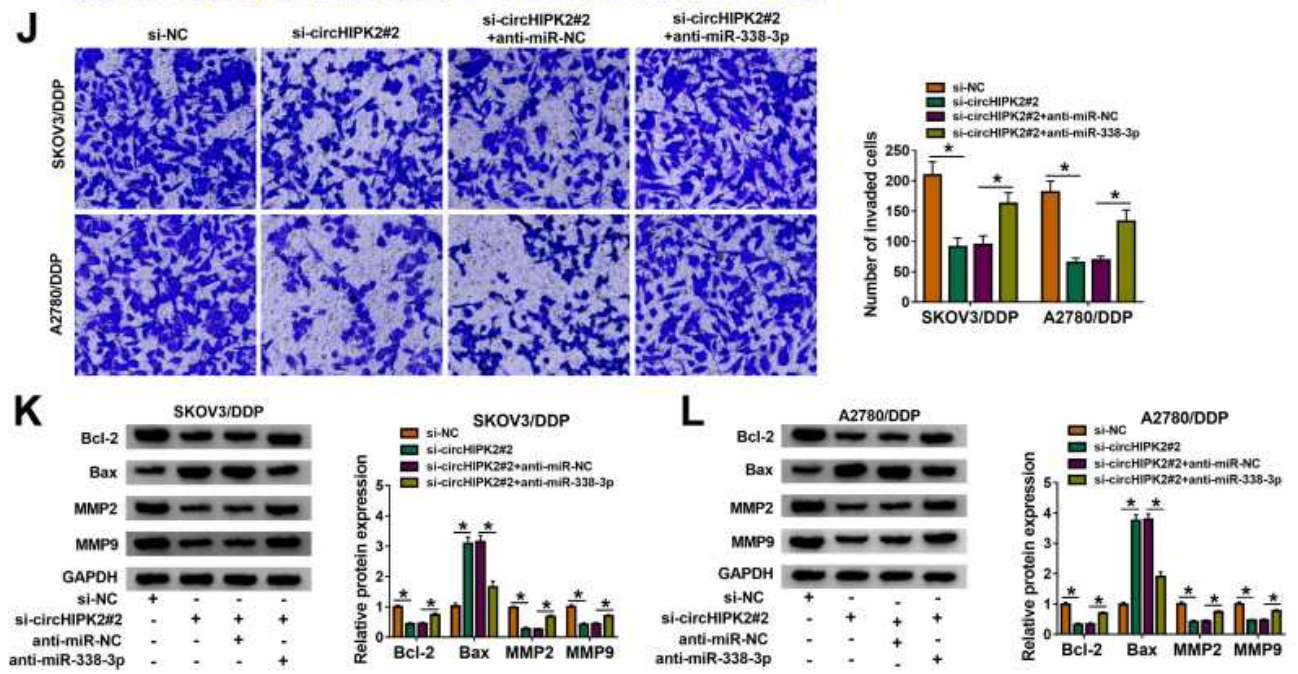

Figure 5 The mutual effect between circHIPK2 and miR-338-3p in DDP-resistant OvCa cells in vitro. SKOV3/DDP and A2780/DDP cells were severally transfected with siNC, si-circHIPK2\#2, si-circHIPK2\#2 along with anti-miR-NC, si-circHIPK2\#2 together with anti-miR-338-3p. (A) RT-qPCR analyzed miR-338-3p expression. (B and C) CCK-8 assay measured IC50 of DDP by monitoring cell viability (\%) after I-I20 $\mu$ M of DDP treatment. (D and E) CCK-8 assay showed OD values at 450 nm after transfection for 0-72 h. (F-L) After transfection for $24 \mathrm{~h},(\mathbf{F}-\mathbf{H})$ FCM method examined (F) apoptosis rate (\%) and $(\mathbf{G}$ and $\mathbf{H})$ cell distribution percentages (\%) in different cell cycle phases, (I and J) transwell assay evaluated numbers of migrated cells and invaded cells, and (K and $\mathbf{L})$ Western blotting detected protein expression of Bcl-2, Bax, MMP2, and MMP9, normalized to GAPDH. ${ }^{* P}<0.05$. Data analysis was performed using two-way ANOVA followed with Tukey's post-hoc analysis. 
A

$$
\begin{aligned}
\text { CHTOP 3' UTR WT } & \text { 5' agAAGAAAUCUGAUUGAUGCUGGa 3' } \\
\text { miR-338-3p } & \text { 3' guUGUUUUAG_UGACUACGACCu 5' } \\
\text { CHTOP 3' UTR MUT } & \text { 5' agCAGGGCGAUGGUCUCCUAGAUa 3' }
\end{aligned}
$$

D

E

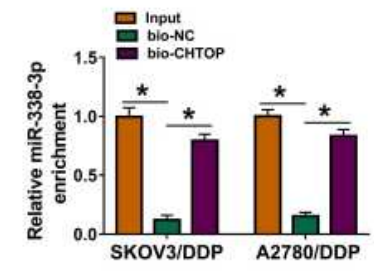

H

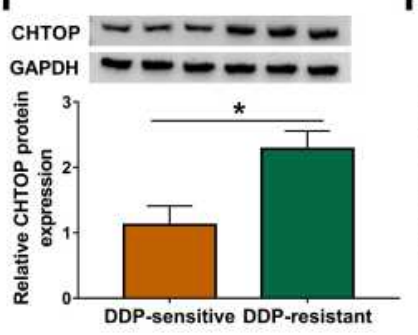

$\mathbf{L}$

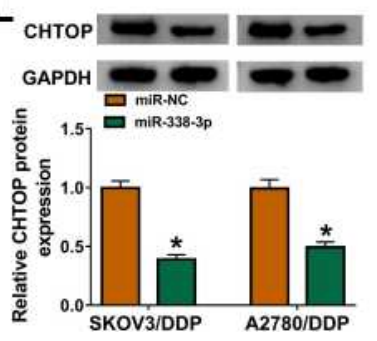

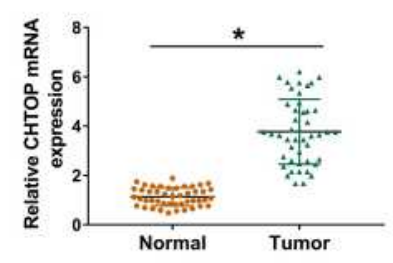

I

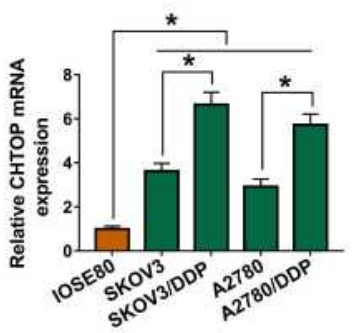

M

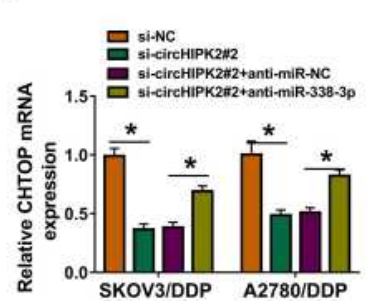

B

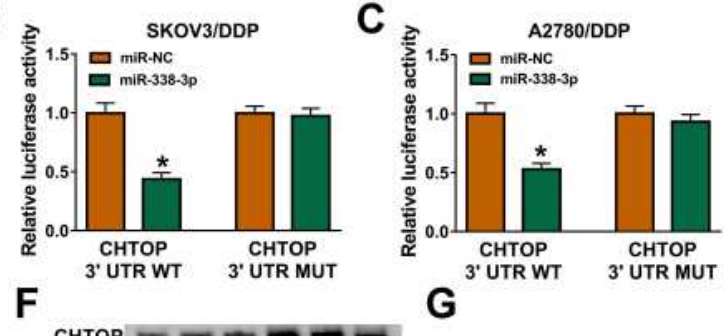

$\mathbf{F}$ GAPDH $=-0-0$
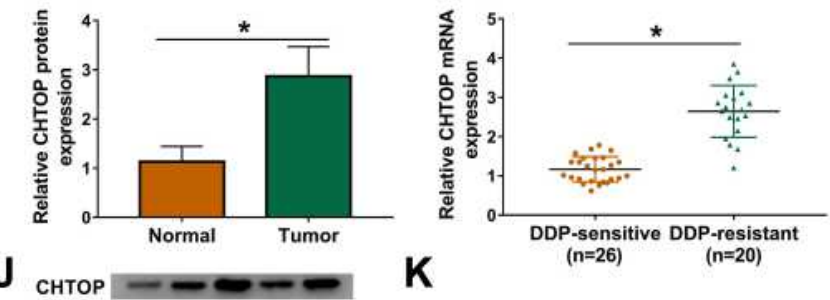
GAPDH $\approx-\infty$
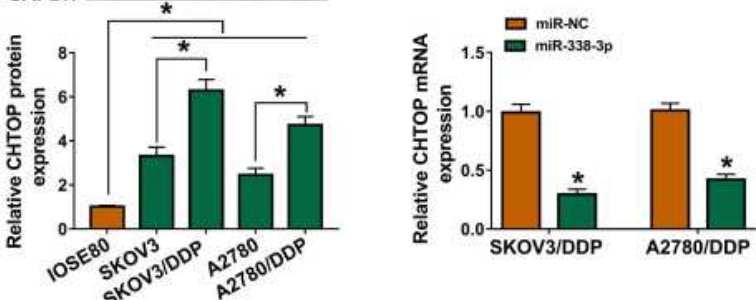

$\mathbf{N}$

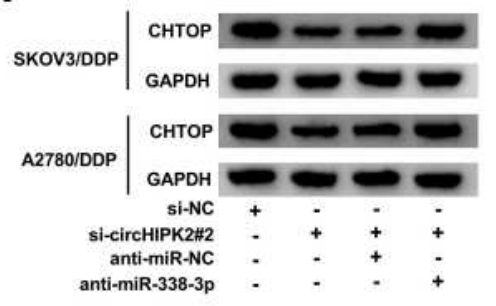

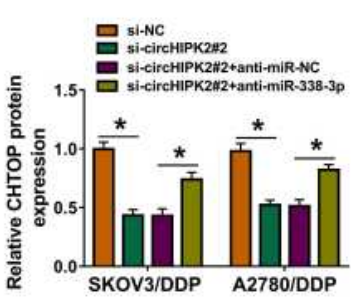

Figure 6 Identification of CHTOP as target gene of miR-338-3p in DDP-resistant OvCa cells. (A) Alignment sequences among CHTOP 3'UTR WT, miR-338-3p and CHTOP 3'UTR MUT. (B and C) Dual-luciferase reporter assay measured luciferase activity of reporter vectors carrying CHTOP 3'UTR WT or MUT in SKOV3/DDP and A2780/DDP cells co-transfected with miR-338-3p or miR-NC. (D) RNA pull-down assay assessed miR-338-3p enrichment by bio-circHIPK2 or bio-NC in SKOV3/DDP and A2780/DDP cells. (E-N) RT-qPCR and Western blotting, respectively, detected CHTOP mRNA expression and CHTOP protein expression in (E and $\mathbf{F})$ OrCa tumor tissues and normal tissues, (G and $\mathbf{H}$ ) DDP-sensitive tumor tissues and DDP-resistant tumor tissues, and (I and J) IOSE80, SKOV3, SKOV3/DDP, A2780, and A2780/DDP cells, as well as (K-N) SKOV3/DDP and A2780/DDP cells severally transfected with miR-NC, miR-338-3p, si-NC, si-circHIPK2\#2, si-circHIPK2\#2 along with anti-miR-NC, si-circHIPK2\#2 together with anti-miR-338-3p. ${ }^{* P}<0.05$. Data analysis was performed using unpaired $t$-test and one-way or two-way ANOVA followed with Tukey's post-hoc analysis.

demonstrated that blocking miR-338-3p could cancel the suppressive role of circHIPK2 knockdown in DDP resistance and malignant behaviors of DDP-resistant OvCa cells in vitro.

\section{CHTOP Served as a Direct Target of miR-338-3p and Was Indirectly Regulated by circHIPK2}

Similarly, CHTOP was predicted to have a complementary binding site of miR-338-3p, and the alignment sequences among CHTOP 3'UTR WT, miR-338-3p and CHTOP
3'UTR MUT were presented (Figure 6A). Overexpression of miR-338-3p attenuated luciferase activity of reporter vector carrying CHTOP 3'UTR WT rather than MUT in SKOV3/ DDP and A2780/DDP cells (Figure 6B and C); enrichment of miR-338-3p could be observed in bio-CHTOP-mediated pulldown contents (Figure 6D). According to above data, there was a direct interaction between CHTOP and miR-338-3p. Expression of CHTOP both at mRNA level and protein level was upregulated in OvCa patients and cells (Figure 6E, F, I and J), and its expression levels were higher in DDPresistant $\mathrm{OvCa}$ tumors and cells (Figure 6G-J). Moreover, CHTOP expression levels were diminished by miR-338-3p 
overexpression via mimic or circHIPK2 silencing via siRNA in SKOV3/DDP and A2780/DDP cells (Figure 6K-N), and CHTOP downregulation in sh-circHIPK2\#2-transfected cells was significantly elevated by silencing miR-338-3p via inhibitor (Figure 6M and N). These data suggested that CHTOP was a target of miR-338-3p and could be indirectly regulated by circHIPK2 via miR-338-3p.

\section{Restoring CHTOP Could Abolish the Suppressive Role of miR-338-3p Overexpression in DDP Resistance and Malignant Behaviors of DDP-Resistant OvCa Cells in vitro}

Overexpression of miR-338-3p resulted in the downregulation of CHTOP protein in SKOV3/DDP and A2780/ DDP cells (Figure 7A), and thus decreased IC50 values of DDP (Figure 7B and C). Proliferation of SKOV3/DDP and A2780/DDP cells was inhibited by upregulating miR338-3p (Figure 7D and E); on the contrary, apoptosis rate was facilitated with miR-338-3p mimic transfection (Figure 7F), accompanied with high Bax expression and low Bcl-2 expression (Figure $7 \mathrm{~K}$ and L). Cell distribution in $\mathrm{G} 0 / \mathrm{G} 1$ phase was increased in miR-338-3poverexpressed SKOV3/DDP and A2780/DDP cells, paralleled with decreased cell distribution in S phase (Figure $7 \mathrm{G}$ and $\mathrm{H}$ ). Suppression of cell migration and invasion was also obtained due to miR-338-3p mimic transfection in SKOV3/DDP and A2780/DDP cells, as evidenced by reduced numbers of transwell migrated cells and invaded cells (Figure 7I and J), and inhibited MMP2 and MMP9 expression (Figure $7 \mathrm{~K}$ and L). These results depicted a suppressive role of miR-338-3p overexpression in DDP resistance and malignant behaviors of DDP-resistant OvCa cells in vitro. Notably, above effects were overall abolished by restoring CHTOP via co-transfection with pEGFP-CHTOP (Figure 7A-L). This outcome supported a reciprocal role between miR-338-3p and CHTOP in DDP-resistant OvCa.

\section{Discussion}

Although advances had been made, platinum resistance remained one of the major obstacle for the poor 5-year survival of OvCa patients at advanced stages. However, it was still lack of an efficient and sensitive method to detect OvCa at early stages. ${ }^{19}$ The expression profile of circRNAs had been identified in five paired DDPsensitive and DDP-resistant tissues of OvCa patients using microarray analysis. ${ }^{20}$ Here, we further investigated the expression and contribution of circHIPK2 in DDPresistant OvCa cells both in vitro and in vivo, as well as the ceRNA pathway.

Several researches had discussed the biological role of circHIPK2 in astrocyte dysfunction and pulmonary fibrosis. $^{21-23}$ In human cancers, circHIPK2 together with other four circRNAs was differentially upregulated in OvCa patients' serum. ${ }^{24}$ Even so, the association between circHIPK2 and tumor development in cancers, especially in OvCa, was left to be elucidated. In this present study, our data revealed an upregulation of circHIPK2 in OvCa tumor tissues and cells, which was consistent with circHIPK2 expression in the serum; ${ }^{24}$ and higher circHIPK2 was discovered in DDP-resistant OvCa tumor tissues and cells, and this finding might be the first evidence between circHIPK2 and DDP resistance. Functionally, blocking circHIPK2 could partially reverse IC50 value of DDP in DDP-resistant OvCa cells, hinting that circHIPK2 knockdown suppressed DDP resistance in OvCa. Moreover, cell proliferation, cell cycle progression, migration and invasion, as well as tumor growth of OvCa cells were overall restrained by silencing circHIPK2, prompting an anti-tumor role of circHIPK2 knockdown in DDP-resistant OvCa cells both in vitro and in vivo.

Mechanically, circHIPK2 showed multiple binding sites of OvCa-related miRNAs, ${ }^{24}$ such as miR-34a and miR-29a. ${ }^{25,26}$ Besides, miR-124 was a confirmed target of circHIPK2 in mouse and human astrocytes. ${ }^{27}$ Here, we predicted that miR-338-3p, miR-584-5p, and miR$889-3 p$ were complementary to circHIPK 2 and could be upregulated in response to circHIPK2 silencing. Among these three miRNAs, miR-338-3p was the most sensitive one in response to circHIPK2 dysregulation, and miR-338$3 p$ was a well-recognized tumor suppressor and regulator of drug resistance ${ }^{28-30}$ including DDP resistance in OvCa. ${ }^{15,16}$ Therefore, this miRNA was selected as the candidate target for circHIPK2. Eventually, we confirmed that miR-338-3p was targeted and sponged by circHIPK2.

In this study, miR-338-3p expression was downregulated in DDP-resistant OvCa tumors and cells, and its overexpression could suppress DDP resistance and malignant behaviors of DDP-resistant OvCa cells in vitro. The inhibitory effects of miR-338-3p on OvCa cell growth, migration and invasion both in vitro and in vivo had been previously demonstrated, as well as DDP resistance. ${ }^{15,16,31}$ In addition, aerobic glycolysis, a hallmark of malignant tumors, in OvCa cells was also affected by restoring miR-338-3p. ${ }^{32}$ Therewith, Zhang et $\mathrm{al}^{33}$ discovered a correlation between miR-338-3p 

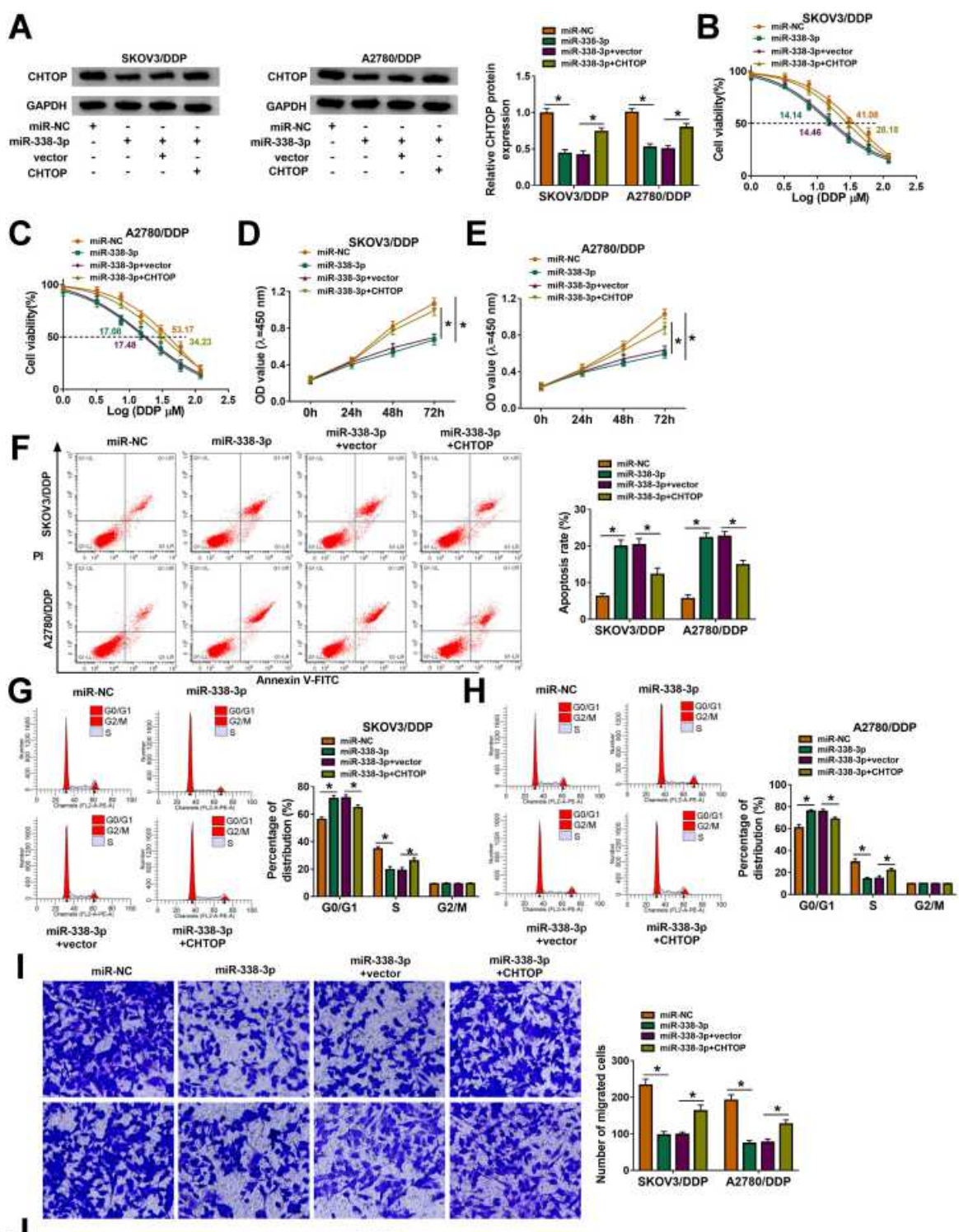

J
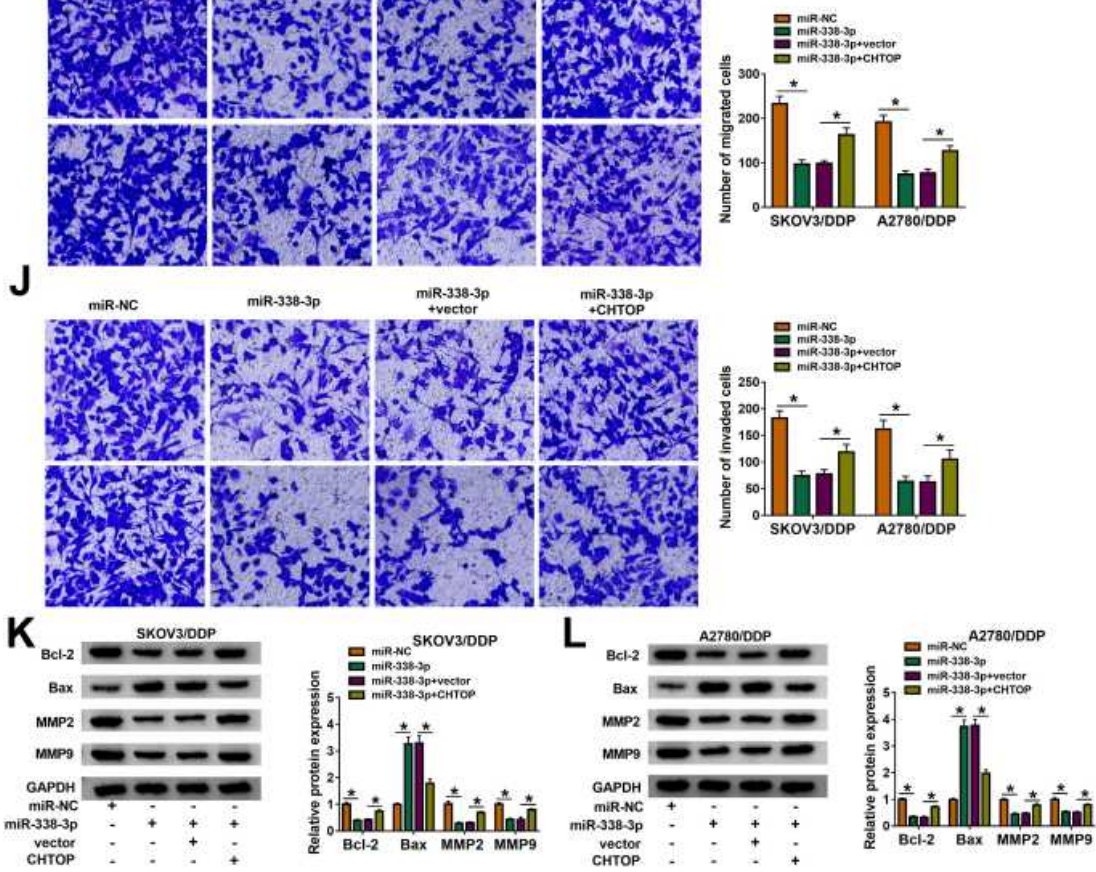

Figure 7 The reciprocal role between CHTOP and miR-338-3p in DDP-resistant OvCa cells in vitro. SKOV3/DDP and A2780/DDP cells were severally transfected with miR-NC, miR-338-3p, miR-338-3p along with pEGFP empty vector (vector), miR-338-3p together with pEGFP-CHTOP vector (CHTOP). (A) Western blotting analyzed CHTOP expression. (B and C) CCK-8 assay measured IC50 of DDP by monitoring cell viability (\%) after I-I20 $\mu$ M of DDP treatment. (D and E) CCK-8 assay showed OD values at $450 \mathrm{~nm}$ after transfection for 0-72 h. (F-L) After transfection for $24 \mathrm{~h},(\mathbf{F}-\mathbf{H})$ FCM method examined $(\mathbf{F})$ apoptosis rate $(\%)$ and $(\mathbf{G}$ and $\mathbf{H})$ cell distribution percentages in different cell cycle phases, ( $\mathbf{I}$ and $\mathbf{J})$ transwell assay evaluated numbers of migrated cells and invaded cells, and ( $\mathbf{K}$ and $\mathbf{L}$ ) Western blotting detected protein expression of Bcl-2, Bax, MMP2, and MMP9, normalized to GAPDH. ${ }^{*}<0.05$. Data analysis was performed using two-way ANOVA followed with Tukey's post-hoc analysis. 


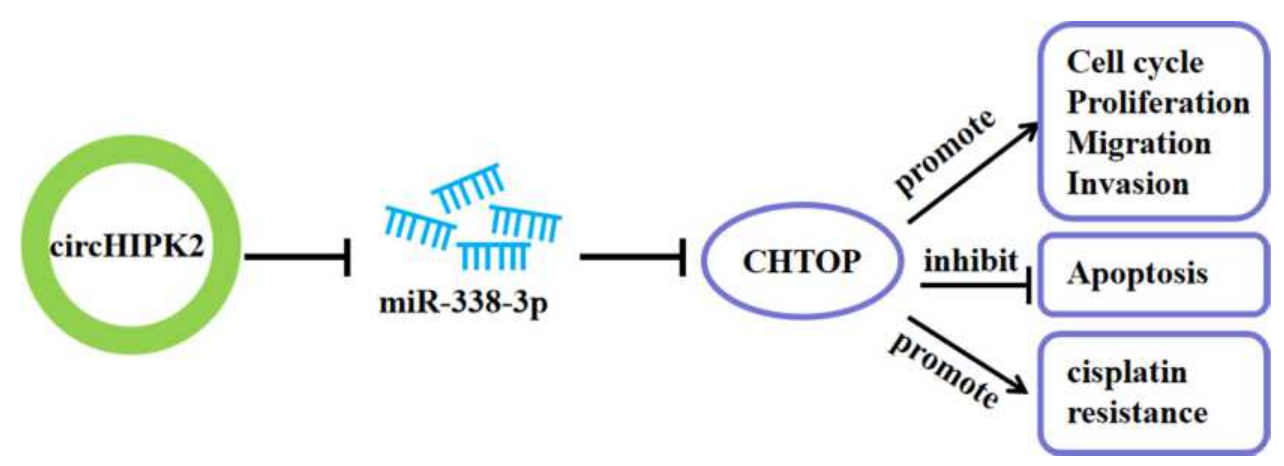

Figure 8 Schematic diagram of circHIPK2 role in OvCa. CircHIPK2/miR-338-3p/CHTOP ceRNA axis could regulate DDP resistance, proliferation, apoptosis, cell cycle progression, migration and invasion of DDP-resistant OvCa cells.

downregulation and recurrence, death or shorter survival rate of OvCa patients. We observed that cell cycle arrest in G0/G1 phase could be induced by overexpressing miR-338-3p, and this finding might be the first-hand document describing the association between miR-338-3p and cells cycle progression of OvCa cells, in spite that it had been widely stated in other human malignant tumors. ${ }^{34-36}$

CHTOP, a versatile regulator of gene-specific transcription and mRNA export, ${ }^{37}$ was a novel modulator in OvCa apoptosis, metastasis, stemness, and DDP resistance. ${ }^{17,18}$ Nevertheless, the miRNA regulation on CHTOP had been little-studied. Here, we identified CHTOP was a downstream target gene of miR-338-3p, and miR-338-3p overexpression downregulated CHTOP to suppress DDP resistance and malignant behaviors of DDP-resistant OvCa cells in vitro. Additionally, restoring CHTOP could elevate IC50 value of DDP, and enhance proliferation, cell cycle progression, migration and invasion of SKOV3/DDP and A2780/DDP cells in spite of miR-338-3p overexpression. Notably, both circHIPK2 knockdown and miR-338-3p upregulation reversed DDP resistance and suppressed malignant behaviors of DDP-resistant OvCa cells through downregulating CHTOP.

In conclusion, this study demonstrated the dysregulation of circHIPK2, miR-338-3p and CHTOP in DDP-resistant OvCa patients' tissues and cells. Elevated circHIPK2 might contribute to DDP resistance and malignant behaviors of DDP-resistant OvCa cells through circHIPK2/miR-338-3p/ CHTOP ceRNA regulatory axis (Figure 8).

\section{Ethics Approval and Consent Participate}

Written informed consent was obtained from patients with approval by the Institutional Review Board in Cancer
Hospital of China Medical University, Liaoning Cancer Hospital \& Institute.

\section{Funding}

This work was supported by Liaoning Provincial Natural Science Foundation Guidance Plan (20180550023).

\section{Disclosure}

The authors declare that they have no conflicts of interest.

\section{References}

1. Reid BM, Permuth JB, Sellers TA. Epidemiology of ovarian cancer: a review. Cancer Biol Med. 2017;14(1):9-32. doi:10.20892/j. issn.2095-3941.2016.0084

2. Bray F, Ferlay J, Soerjomataram I, Siegel RL, Torre LA, Jemal A. Global cancer statistics 2018: GLOBOCAN estimates of incidence and mortality worldwide for 36 cancers in 185 countries. $C A$ Cancer J Clin. 2018;68(6):394-424. doi:10.3322/caac.21492

3. Kossai M, Leary A, Scoazec JY, Genestie C. Ovarian cancer: a heterogeneous disease. Pathobiology. 2018;85(1-2):41-49. doi:10.1159/000479006

4. Markman M. Pharmaceutical management of ovarian cancer: current status. Drugs. 2019;79(11):1231-1239. doi:10.1007/s40265-01901158-1

5. Damia G, Broggini M. Platinum resistance in ovarian cancer: role of DNA repair. Cancers. 2019;11(1):119. doi:10.3390/cancers11010119

6. Lokadasan R, James FV, Narayanan G, Prabhakaran PK. Targeted agents in epithelial ovarian cancer: review on emerging therapies and future developments. Ecancermedicalscience. 2016;10:626. doi:10.3332/ecancer.2016.626

7. Hu Y, Zhu QN, Deng JL, Li ZX, Wang G, Zhu YS. Emerging role of long non-coding RNAs in cisplatin resistance. Onco Targets Ther. 2018;11:3185-3194. doi:10.2147/OTT.S158104

8. Yu X, Zheng H, Chan MT, Wu WK. Modulation of chemoresponsiveness to platinum-based agents by microRNAs in cancer. $\mathrm{Am}$ J Cancer Res. 2017;7(9):1769-1778.

9. Song L, Cui Z, Guo X. Comprehensive analysis of circular RNA expression profiles in cisplatin-resistant non-small cell lung cancer cell lines. Acta Biochim Biophys Sin (Shanghai). 2020;52 (9):944-953. doi:10.1093/abbs/gmaa085

10. Kristensen LS, Andersen MS, Stagsted LVW, Ebbesen KK, Hansen TB, Kjems J. The biogenesis, biology and characterization of circular RNAs. Nat Rev Genet. 2019;20(11):675-691. doi:10.1038/ s41576-019-0158-7 
11. Dong P, Xu D, Xiong Y, et al. The expression, functions and mechanisms of circular RNAs in gynecological cancers. Cancers. 2020;12 (6):1472. doi:10.3390/cancers12061472

12. Kuwano Y, Nishida K, Akaike Y, et al. Homeodomain-interacting protein kinase-2: a critical regulator of the DNA damage response and the epigenome. Int J Mol Sci. 2016;17(10):1638. doi:10.3390/ ijms17101638

13. Xu T, Wang M, Jiang L, et al. CircRNAs in anticancer drug resistance: recent advances and future potential. Mol Cancer. 2020;19 (1):127. doi:10.1186/s12943-020-01240-3

14. Yang Y, Ren J, Huang Q, et al. CircRNA expression profiles and the potential role of CircZFP644 in mice with severe acute pancreatitis via sponging miR-21-3p. Front Genet. 2020;11:206. doi:10.3389/ fgene. 2020.00206

15. Zhang R, Shi H, Ren F, et al. MicroRNA-338-3p suppresses ovarian cancer cells growth and metastasis: implication of Wnt/catenin beta and MEK/ERK signaling pathways. J Exp Clin Cancer Res. 2019;38 (1):494. doi:10.1186/s13046-019-1494-3

16. Niu Q, Liu Z, Gao J, Wang Q. MiR-338-3p enhances ovarian cancer cell sensitivity to cisplatin by downregulating WNT2B. Yonsei Med J. 2019;60(12):1146-1156. doi:10.3349/ymj.2019.60.12.1146

17. Feng X, Bai X, Ni J, et al. CHTOP in chemoresistant epithelial ovarian cancer: a novel and potential therapeutic target. Front Oncol. 2019;9:557. doi:10.3389/fonc.2019.00557

18. Feng X, Li L, Wang L, Luo S, Bai X. Chromatin target of protein arginine methyltransferase regulates invasion, chemoresistance, and stemness in epithelial ovarian cancer. Biosci Rep. 2019;39(4). doi:10.1042/BSR20190016

19. Alshamrani AA. Roles of microRNAs in ovarian cancer tumorigenesis: two decades later, what have we learned? Front Oncol. 2020;10:1084. doi:10.3389/fonc.2020.01084

20. Zhao Z, Ji M, Wang Q, He N, Li Y. Circular RNA Cdrlas upregulates SCAI to suppress cisplatin resistance in ovarian cancer via miR-1270 suppression. Mol Ther Nucleic Acids. 2019;18:24-33. doi:10.1016/j. omtn.2019.07.012

21. Cao Z, Xiao Q, Dai X, et al. circHIPK2-mediated sigma-1R promotes endoplasmic reticulum stress in human pulmonary fibroblasts exposed to silica. Cell Death Dis. 2017;8(12):3212. doi:10.1038/ s41419-017-0017-4

22. Wang G, Han B, Shen L, et al. Silencing of circular RNA HIPK2 in neural stem cells enhances functional recovery following ischaemic stroke. EBioMedicine. 2020;52:102660. doi:10.1016/j. ebiom.2020.102660

23. Zhang Y, Huang $R$, Cheng $M$, et al. Gut microbiota from NLRP3-deficient mice ameliorates depressive-like behaviors by regulating astrocyte dysfunction via circHIPK2. Microbiome. 2019;7 (1):116. doi:10.1186/s40168-019-0733-3

24. Wang J, Wu A, Yang B, Zhu X, Teng Y, Ai Z. Profiling and bioinformatics analyses reveal differential circular RNA expression in ovarian cancer. Gene. 2020;724:144150. doi:10.1016/j. gene. 2019.144150

OncoTargets and Therapy

\section{Publish your work in this journal}

OncoTargets and Therapy is an international, peer-reviewed, open access journal focusing on the pathological basis of all cancers, potential targets for therapy and treatment protocols employed to improve the management of cancer patients. The journal also focuses on the impact of management programs and new therapeutic

Submit your manuscript here: https://www.dovepress.com/oncotargets-and-therapy-journal
25. Welponer H, Tsibulak I, Wieser V, et al. The miR-34 family and its clinical significance in ovarian cancer. $J$ Cancer. 2020;11 (6): 1446-1456. doi:10.7150/jca.33831

26. Yu PN, Yan MD, Lai HC, et al. Downregulation of miR-29 contributes to cisplatin resistance of ovarian cancer cells. Int $J$ Cancer. 2014;134(3):542-551. doi:10.1002/ijc.28399

27. Huang R, Zhang Y, Han B, et al. Circular RNA HIPK2 regulates astrocyte activation via cooperation of autophagy and ER stress by targeting MIR124-2HG. Autophagy. 2017;13(10):1722-1741. doi:10.1080/15548627.2017.1356975

28. Han J, Li J, Tang K, et al. miR-338-3p confers 5-fluorouracil resistance in p53 mutant colon cancer cells by targeting the mammalian target of rapamycin. Exp Cell Res. 2017;360(2):328-336. doi:10.1016/j.yexcr.2017.09.023

29. Shan Y, Li X, You B, Shi S, Zhang Q, You Y. MicroRNA-338 inhibits migration and proliferation by targeting hypoxia-induced factor 1alpha in nasopharyngeal carcinoma. Oncol Rep. 2015;34 (4):1943-1952. doi:10.3892/or.2015.4195

30. Xu H, Zhao L, Fang Q, et al. MiR-338-3p inhibits hepatocarcinoma cells and sensitizes these cells to sorafenib by targeting hypoxia-induced factor 1alpha. PLoS One. 2014;9(12):e115565. doi:10.1371/journal.pone. 0115565

31. Liu X, Wen J, Wang H, Wang Y. Long non-coding RNA LINC00460 promotes epithelial ovarian cancer progression by regulating microRNA-338-3p. Biomed Pharmacother. 2018;108:1022-1028. doi:10.1016/j.biopha.2018.09.103

32. Zhang Y, Shi B, Chen J, Hu L, Zhao C. MiR-338-3p targets pyruvate kinase M2 and affects cell proliferation and metabolism of ovarian cancer. Am J Transl Res. 2016;8(7):3266-3273.

33. Zhang R, Shi H, Ren F, et al. Down-regulation of miR-338-3p and up-regulation of MACC1 indicated poor prognosis of epithelial ovarian cancer patients. J Cancer. 2019;10(6):1385-1392. doi:10.7150/ jca. 29502

34. Cao R, Shao J, Hu Y, et al. microRNA-338-3p inhibits proliferation, migration, invasion, and EMT in osteosarcoma cells by targeting activator of $90 \mathrm{kDa}$ heat shock protein ATPase homolog 1. Cancer Cell Int. 2018;18(1):49. doi:10.1186/s12935-018-0551-x

35. Zhang P, Shao G, Lin X, Liu Y, Yang Z. MiR-338-3p inhibits the growth and invasion of non-small cell lung cancer cells by targeting IRS2. Am J Cancer Res. 2017;7(1):53-63.

36. Guo B, Liu L, Yao J, et al. miR-338-3p suppresses gastric cancer progression through a PTEN-AKT axis by targeting P-REX2a. Mol Cancer Res. 2014;12(3):313-321. doi:10.1158/1541-7786.MCR-130507

37. Izumikawa K, Ishikawa H, Simpson RJ, Takahashi N. Modulating the expression of Chtop, a versatile regulator of gene-specific transcription and mRNA export. RNA Biol. 2018;15(7):849-855. doi:10.1080/ 15476286.2018 .1465795 agents and protocols on patient perspectives such as quality of life, adherence and satisfaction. The manuscript management system is completely online and includes a very quick and fair peer-review system, which is all easy to use. Visit http://www.dovepress.com/ testimonials.php to read real quotes from published authors. 